\title{
$\Upsilon$ production at the Tevatron and the LHC
}

\author{
P. Artoisenet ${ }^{a}$, J. Campbell ${ }^{b}$, J.P. Lansberg ${ }^{c}$, F. Maltoni ${ }^{a}$, F. Tramontano ${ }^{d}$ \\ ${ }^{a}$ Center for Particle Physics and Phenomenology (CP3), \\ Université catholique de Louvain, B-1348 Louvain-la-Neuve, Belgium \\ ${ }^{b}$ Department of Physics and Astronomy, University of Glasgow, Glasgow G12 8QQ, United Kingdom \\ ${ }^{c}$ Institut für Theoretische Physik, Universität Heidelberg, D-69120 Heidelberg, Germany \\ ${ }^{d}$ Università di Napoli Federico II, Dipartimento di Scienze Fisiche, \\ and INFN, Sezione di Napoli, I-80126 Napoli, Italy
}

\begin{abstract}
We update the theoretical predictions for direct $\Upsilon(n S)$ hadroproduction in the framework of NRQCD. We show that the next-to-leading order corrections in $\alpha_{S}$ to the color-singlet transition significantly raise the differential cross section at high $p_{T}$ and substantially affect the polarization of the $\Upsilon$. Motivated by the remaining gap between the NLO yield and the cross section measurements at the Tevatron, we evaluate the leading part of the $\alpha_{S}^{5}$ contributions, namely those coming from $\Upsilon(n S)$ associated with three light partons. The differential color-singlet cross section at $\alpha_{S}^{5}$ is in substantial agreement with the data, so that there is no evidence for the need of color-octet contributions. Furthermore, we find that the polarization of the $\Upsilon(n S)$ is longitudinal. We also present our predictions for $\Upsilon(n S)$ production at the LHC.
\end{abstract}

PACS numbers: 12.38.Bx,14.40.Gx,13.85.Ni

Even though the discrepancy between experimental measurements and theoretical predictions for $\Upsilon(n S)$ hadroproduction is less dramatic than for $J / \psi$ and $\psi^{\prime}$, it is still unclear which are the dominant mechanisms at work in those processes [1]. The leading order (LO) $\alpha_{S}^{3}$ result [2] in the $v$ expansion of non-relativistic QCD (NRQCD) [3] neither predicts the yield seen in the data [4, 5], undershooting it by a factor of ten at $p_{T}$ around $20 \mathrm{GeV}$, nor shows the correct $p_{T}$ behavior. In this case it has been shown that the inclusion of higher- $v$ corrections, in particular those associated with the coloroctet mechanism, allows the data to be described. The yield and the $p_{T}$ shape can be reproduced, at the cost of introducing two unknown long-distance-matrix elements of NRQCD that can be determined from fits to the data [6]. However, the polarization predicted following this approach disagrees with both the published $\mathrm{CDF}[5]$ and new preliminary $\mathrm{D} \emptyset$ measurements [7].

In view of such a puzzling situation, it is worth reexamining in detail the available theoretical predictions. In Refs. [8, 9], the complete set of $\alpha_{S}^{4}$ corrections to colorsinglet production was calculated for the first time. The results confirmed the importance of the new channels that open up at this order, which is due to their different $p_{T}$ scaling (see Fig. 1] (c,d)). In this Letter we apply those results to a phenomenological study of $\Upsilon$ production at the Tevatron, comparing the differential cross section at next-to-leading order (NLO) with the data from the CDF collaboration. We also argue that some $\alpha_{S}^{5}$ contributions coming from three-jet configurations, i.e. $\Upsilon+j j j$, such as those arising from gluon-fragmentation (Fig. 1 (e)) and "high-energy enhanced" (Fig. 1 (f)) channels are important. We show that by adding such $\alpha_{S}^{5}$ contributions to the NLO yield, the experimental measurements are reproduced. We also predict the polarization of the $\Upsilon$ from the angular distribution of the leptons produced in the decay.

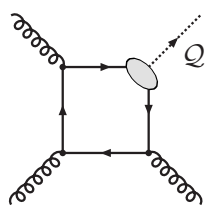

(a)

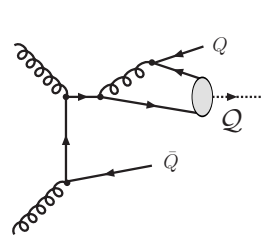

(d)

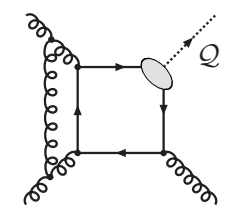

(b)

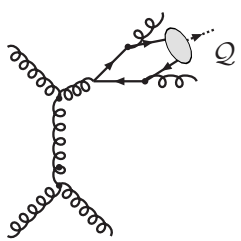

(e)

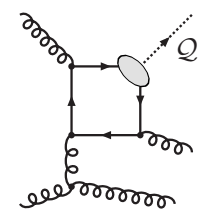

(c)

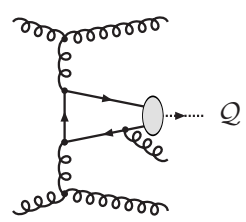

(f)
FIG. 1: Representive diagrams contributing to $\Upsilon$ hadroproduction at orders $\alpha_{S}^{3}(\mathrm{a}), \alpha_{S}^{4}(\mathrm{~b}, \mathrm{c}, \mathrm{d}), \alpha_{S}^{5}$ (e,f). See discussions in the text.

We first present the NLO predictions for the $p_{T}$ spectrum of the $\Upsilon$ at leading order in $v$, following the same procedure as in Ref. [8] and adding information on the polarization of the quarkonium state. In our numerical evaluation of the differential cross section and polarization parameters, we have used: $\left|R_{\Upsilon(1 S)}(0)\right|^{2}=$ $6.48 \mathrm{GeV}^{3}$ and $\left|R_{\Upsilon(3 S)}(0)\right|^{2}=2.47 \mathrm{GeV}^{3}[1] ; \mu_{0}=$ $\sqrt{\left(2 m_{b}\right)^{2}+p_{T}^{2}} ; \operatorname{Br}\left(\Upsilon(1 S) \rightarrow \mu^{+} \mu^{-}\right)=0.0248$ and $\operatorname{Br}\left(\Upsilon(3 S) \rightarrow \mu^{+} \mu^{-}\right)=0.0218 ; m_{b}=4.75 \mathrm{GeV} ; \mathrm{PDF}$ set: CTEQ6M [10]. For comparison, we also plot the LO yield, for which we used the PDF set CTEQ6L1. 
The experimental results for the cross section differential in $p_{T}$ for prompt $\Upsilon(1 S)$ and $\Upsilon(2 S)$, as well as the cross section for direct $\Upsilon(3 S)$ and the polarization for prompt $\Upsilon(1 S)$ were obtained in Run I at $\sqrt{s}=1.8$ $\mathrm{TeV}$ [5]. For Run II at $\sqrt{s}=1.96 \mathrm{TeV}$, so far only $\mathrm{D} \emptyset$ have analyzed data for $\Upsilon(1 S)$ [1]. The comparison performed in [11] shows full agreement with [5], once the difference in $\sqrt{s}$ is taken into account.

In order to compare our calculation for direct $\Upsilon(1 S)$ production with the CDF data, we have multiplied the most recent prompt- $\Upsilon(1 S)$ cross-section measurement of [5] by the direct fraction $F_{\text {direct }}^{\Upsilon(1 S)}=0.5 \pm 0.12$. This fraction was obtained from an older sample [4, where the selection cuts $\left|p_{T}\right|>8 \mathrm{GeV}$ and $|\eta|<0.7$ were applied. The similar $p_{T}$ dependence observed for prompt$\Upsilon(1 S)$ and direct- $\Upsilon(3 S)$ production, as shown in Fig. 2 of [5], confirms that this fraction should not depend very strongly on $p_{T}$. This justifies using the same fraction for the whole $p_{T}$ range. The errors from the prompt cross section and the direct fraction have been combined in quadrature. Similar information on the direct yield of $\Upsilon(2 S)$ is not yet available.

Our results for direct production of $\Upsilon(1 S)$ and $\Upsilon(3 S)$ at $\sqrt{s}=1.8 \mathrm{TeV}$ are shown in Fig. 2. The curves labeled as $\mathrm{NNLO}^{\star}$ will be discussed later on. In order to estimate the theoretical uncertainties, we have varied the renormalization and factorization scales between $0.5 \mu_{0}$ and $2 \mu_{0}$, keeping them equal. We have combined the resulting uncertainties in quadrature with those resulting from variation of the bottom quark mass, $m_{b}=4.75 \pm 0.25 \mathrm{GeV}$. The uncertainty associated with the non-perturbative parameter $\left|R_{\Upsilon(n S)}(0)\right|^{2}$ [6, 12, corresponding to an overall normalization, is not included. The curves for direct $\Upsilon(2 S)$ production can be easily obtained - at this order in $v$ - by adopting the corresponding values for the leptonic branching ratio and for the non-perturbative matrix element, $\left|R_{\Upsilon(2 S)}(0)\right|^{2}$.

As a first comment, we note that the LO and NLO results are very similar at low $p_{T}$, i.e. where the bulk of the cross section is found. This implies that the $\alpha_{S}^{4}$ corrections are small in this region and that the perturbative expansion of the total cross section is under control. On the other hand, the relative importance of the NLO corrections dramatically increases at larger $p_{T}$, where new $p_{T}^{-6}$ channels (Fig. 1 (c)) start to dominate. This behavior significantly reduces the discrepancy with the experimental data that is found at LO. However, the NLO prediction still drops too fast in comparison with the data. Note that the NLO calculation also takes into account associated production $\Upsilon+b \bar{b}$ (Fig. 1](d)). This contribution, in spite of reproducing the shape of the data very well at both low and high $p_{T}$ (asymptotically it behaves as $p_{T}^{-4}$ ), remains negligible up to at least $p_{T}=30 \mathrm{GeV}$.

Throughout our calculation, the polarization information of the $\Upsilon$ can be traced through the angular distributions of the two decay leptons. Defining $\theta$ as the angle
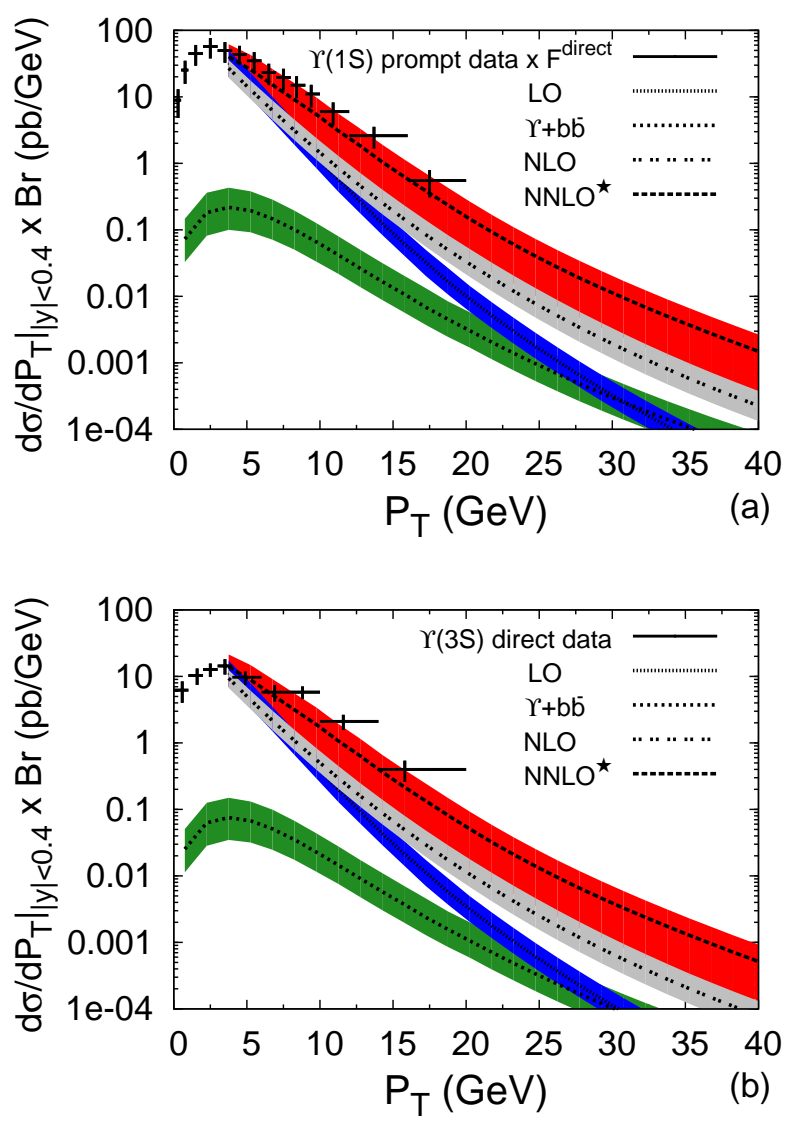

FIG. 2: Results for (a) $\Upsilon(1 S)+X$ and (b) $\Upsilon(3 S)+X$ at LO $\left(\alpha_{S}^{3}\right)$, for associated production $\left(\alpha_{S}^{4}\right)$, for full NLO $\left(\alpha_{S}^{3}+\alpha_{S}^{4}\right)$ and for $\mathrm{NNLO}^{\star}$ (up to $\alpha_{S}^{5}$ ) compared with the direct yield at $\sqrt{s}=1.8 \mathrm{TeV}$ measured by the CDF collaboration [5]. The theoretical-error bands for LO, NLO and associated production come from combining the uncertainties resulting from the choice of $\mu_{f}, \mu_{r}, m_{b}$. The uncertainty of $\mathrm{NNLO}^{\star}$ includes the variation of the cutoff, $s_{i j}^{\min }$, between $0.5 m_{b}^{2}$ and $2 m_{b}^{2}$ and of the scales $\mu_{r}$ and $\mu_{f}$.

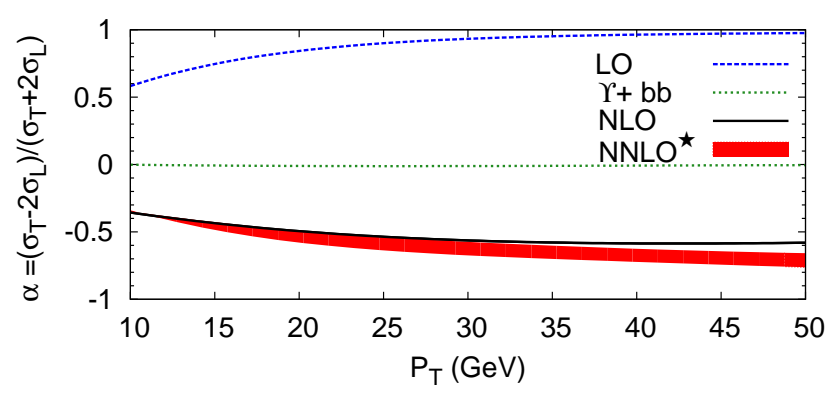

FIG. 3: Polarization for direct $\Upsilon(n S)(n=1,2,3)$ production at $\sqrt{s}=1.8 \mathrm{TeV}$ up to the order $\alpha_{S}^{5}\left(\mathrm{NNLO}^{\star}\right)$. Most of the uncertainties on $\alpha$ for LO, associated production and NLO cancel. The uncertainty band of the $\left(\mathrm{NNLO}^{\star}\right)$ result comes from the variation of the cutoff $s_{i j}^{\mathrm{min}}$. 
between the $\ell^{+}$direction in the quarkonium rest frame and the quarkonium direction in the laboratory frame, the normalized angular distribution $I(\cos \theta)$ reads

$$
I(\cos \theta)=\frac{3}{2(\alpha+3)}\left(1+\alpha \cos ^{2} \theta\right),
$$

from which we have extracted the polarization parameter $\alpha$ bin by bin in $p_{T}$. Our results are shown in Fig. 3 along with the curves for the LO yield and for the associated production alone. Even though the inclusion of NLO corrections brings the predictions closer to the prompt data, the discrepancy in the normalization makes such a comparison not very compelling.

As already noted, the discrepancy between the data and the full NLO (up to $\alpha_{S}^{4}$ ) result in Fig. 2 increases with $p_{T}$. This might be taken as an indication that one or more processes with a more gentle $p_{T}$ scaling than the $\alpha_{S}^{4}$ contributions, which scale at most as $p_{T}^{-6}$ (disregarding the sub-dominant $\Upsilon+b \bar{b}$ channel), are still missing. In this section, we argue that the $\alpha_{S}^{5}$ terms provide us with the missing contributions. Indeed, the gluon fragmentation channel (Fig. 1 (e)) occurs for the first time at order $\alpha_{S}^{5}$ and behaves like $p_{T}^{-4}$ at large transverse momentum. At next-to-next-to-leading order (NNLO) "high-energy enhanced" channels (Fig. 1 (f)) also appear for the first time, whose contributions have previously been approximated in the $k_{T}$ factorization approach [13]. Both these contributions, which exhaust the possibilities of new kinematical enhancements at higher order, are at the Born level and therefore finite at $\alpha_{S}^{5}$. They are a subset of $p \bar{p} \rightarrow \Upsilon+j j j$ ( $j$ stands for $u, d, s, c, g$ ) and provide us with new mechanisms to produce a high- $p_{T} \Upsilon$ with a lower kinematic suppression. They can therefore be expected to dominate the differential cross section at NNLO accuracy in the region of large transverse momentum.

We present here an estimate of these dominant $\alpha_{S}^{5}$ contributions at large $p_{T}$, bypassing the presently outof-reach inclusive calculation of $p \bar{p} \rightarrow \Upsilon+X$ at NNLO accuracy. We consider the whole set of $\alpha_{S}^{5}$ processes contributing to the color-singlet production of a $\Upsilon$ associated with three light partons. The computation of the associated matrix elements is technically possible via the approach presented in Ref. 14. In order to protect the integrated cross section from soft and collinear divergences, we impose a "democratic" invariant-mass cut $s_{i j}^{\min }$ of the order of $m_{b}^{2}$ to any pair of light partons so that the phase-space integration remains finite. For new channels opening up at $\alpha_{S}^{5}$, the dependence on the value of $s_{i j}^{\min }$ is expected to be small in the region $p_{T} \gg m_{b}$, since no collinear or soft divergences can appear there. For channels whose Born-level contribution occurs at $\alpha_{S}^{3}$ or $\alpha_{S}^{4}$, the invariant-mass cut results in potentially large logarithms of $s_{i j} / s_{i j}^{\min }$ in the differential cross sections. However, these logs factorize over lower order amplitudes that are suppressed by powers of $p_{T}$ compared to the dominant $\alpha_{S}^{5}$ contributions. The result is that the sensitivity to the value chosen for the minimal invariant mass cut $s_{i j}^{\min }$ is expected to die away as $p_{T}$ increases.

In fact, this argument can be tested by using it to estimate the dominant contribution to the production of $\Upsilon$ at NLO accuracy. The differential cross section for the real $\alpha_{s}^{4}$ corrections, $\Upsilon+j j$ production, is displayed in Fig. 4 The grey band is obtained by varying the invariant-mass cut $s_{i j}^{\min }$ between any pairs of light partons from $0.5 m_{b}^{2}$ to $2 m_{b}^{2}$. The yield becomes insensitive to the value of $s_{i j}^{\min }$ as $p_{T}$ increases, and it reproduces very accurately the differential cross section at NLO accuracy.

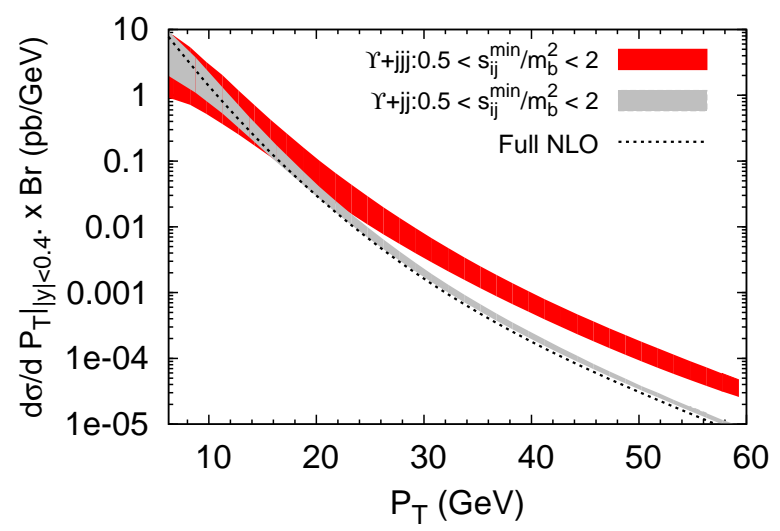

FIG. 4: Full computation at NLO for $\Upsilon+X$ (dashed line) vs. $\Upsilon(1 S)+2$ light partons with a cut on $s_{i j}^{\min }$ (grey band). The red band shows the cross section for $\Upsilon(1 S)+3$ light partons with the same cut. (see text for details).

The computation of $p \bar{p} \rightarrow \Upsilon+j j j$ at tree level is in principle straightforward, but technically quite challenging: several parton-level subprocesses contribute, each involving up to hundreds of Feynman diagrams. We follow the approach described in Ref. [14, which allows the automatic generation of both the subprocesses and the corresponding scattering amplitudes. We find that the subprocess $g g \rightarrow \Upsilon+g g g$ dominates, providing $50 \%$ of the whole yield. The uncertainty associated with the choice of the cut $s_{i j}^{\min }$ is substantial although, as can be seen in Fig. 4, it decreases at large $p_{T}$ where these contributions are dominant.

The differential cross-sections for $\Upsilon(1 S)$ and $\Upsilon(3 S)$ are shown in Fig. 2. The red band (referred to as $\mathrm{NNLO}^{\star}$ ) corresponds to the sum of the NLO yield and the $\Upsilon+j j j$ contributions. The contribution of $\Upsilon$ production with three light partons fills the gap between the data and the NLO calculation. The $\alpha_{S}^{5}$ contribution is very sensitive to the choice of the renormalization scale, $\mu_{r}$. This is expected: for moderate values of the $p_{T}$, the missing virtual part might be important, whereas at large $p_{T}$, the yield is dominated by Born-level $\alpha_{S}^{5}$-channels from which we expect a large dependence on $\mu_{r}$. Even though the uncertainty on the normalization is rather large, the pre- 
diction of the $p_{T}$ shape is quite robust and agrees well with the behavior found in the data. At leading order in $v$, results for $\Upsilon(2 S)$ can be obtained simply by changing $\left|R_{\Upsilon(n S)}(0)\right|^{2}$ and the branching ratio. The predictions for the polarization parameter $\alpha$ are only slightly affected by the $\mathrm{NNLO}^{\star}$ contributions and remain negative at high transverse momentum, Fig. 3. Finally, we show our predictions for the $p_{T}$ distributions at the LHC $(\sqrt{s}=14$ $\mathrm{TeV}$ ) in Fig. 5 .

Obviously, our analysis cannot be extended to rather low $p_{T}$, where the approximations on which it is based no longer hold. In this case, one could improve the predictions by merging the matrix elements with parton showers using one of the approaches available in the literature [15], or by performing an analytic resummation [16].

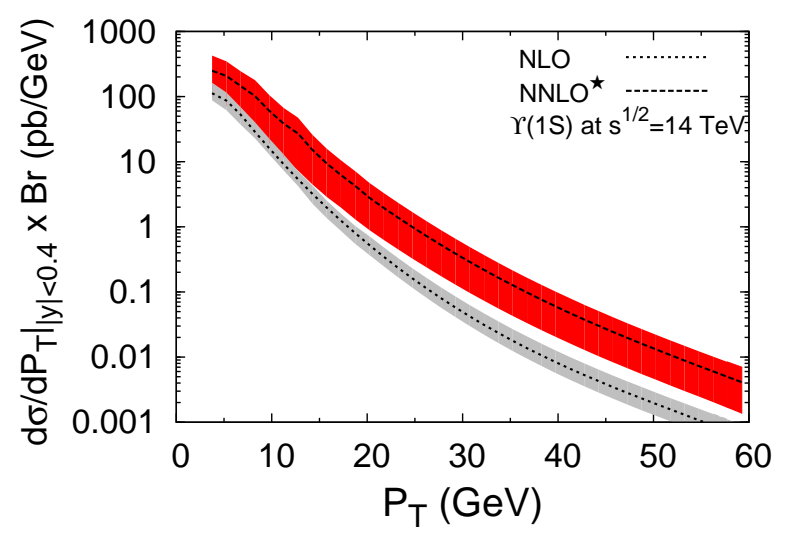

FIG. 5: $\Upsilon(1 S)+X$ at $\sqrt{s}=14 \mathrm{TeV}$. Same parameters as for Fig 2. Results for the $\Upsilon(3 S)+X$ can be obtained by rescaling the curves by the ratio of the corresponding wave functions values at the origin and branching ratios.

In conclusion, we have compared the full $\mathrm{NLO}\left(\alpha_{S}^{4}\right)$ cross section for polarized direct hadroproduction of $\Upsilon(n S)$ to the data from the Tevatron. At this order, the polarization parameter $\alpha$ is negative and decreases with $p_{T}$. However, the yield is still too low compared to the data especially at high $p_{T}$.

This led us to consider a subset of the NNLO $\left(\alpha_{S}^{5}\right)$ corrections, namely those corresponding to the production of a $\Upsilon$ associated with three light partons and containing $p_{T}^{-4}$ contributions. Integrating the amplitudes for such processes at $\alpha_{S}^{5}$ leads to divergences in some phase-space regions that we avoided by imposing a minimal invariant mass between the light partons. This was motivated by the fact that the neglected contributions are proportional to $\alpha_{S}^{4}$ amplitudes, which scale at most as $p_{T}^{-6}$, so that we expected this approach to give a reliable estimate of the full $\alpha_{S}^{5}$ result at large $p_{T}$. We validated our expectations by comparing such an approach with the computation at $\alpha_{S}^{4}$ and by explicitly checking the sensitivity of our results to the cutoff choice at high $p_{T}$. The estimated
$\alpha_{S}^{5}$-contributions appear to fill the gap between the full NLO $\left(\alpha_{S}^{4}\right)$ and the data.

As a further result, we have predicted the polarization parameter $\alpha$ to be negative and only slightly decrease with $p_{T}$, indicating a longitudinally polarized yield. More accurate data, in particular for $\Upsilon(3 S)$ and possibly at higher $p_{T}$, are needed to check these predictions.

In summary, the comparison with the available data suggests that there is no need to include contributions from color-octet transitions for $\Upsilon(n S)$ production. For the $\psi$ case, the situation is less clear since the addition of higher-order QCD corrections seems not to suffice to describe the data [8, 17, 18. In this case, other mechanisms may be at work such as those recently discussed in Refs. [19, 20].

[1] J. P. Lansberg, Int. J. Mod. Phys. A 21 (2006) 3857; N. Brambilla et al., CERN 2005-005, hep-ph/0412158, M. Kramer, Prog. Part. Nucl. Phys. 47 (2001) 141.

[2] C-H. Chang, Nucl. Phys. B 172 (1980) 425; R. Baier and R. Rückl, Phys. Lett. B 102 (1981) 364; Z. Phys. C 19 (1983) 251; E. L. Berger and D. L. Jones, Phys. Lett. B 121 (1983) 61.

[3] G. T. Bodwin, E. Braaten and G. P. Lepage, Phys. Rev. D 51, 1125 (1995) [Erratum-ibid. D 55, 5853 (1997)]

[4] A. A. Affolder et al. [CDF Collaboration], Phys. Rev. Lett. 84 (2000) 2094

[5] D. Acosta et al. [CDF Collaboration], Phys. Rev. Lett. 88 (2002) 161802.

[6] E. Braaten, S. Fleming and A. K. Leibovich, Phys. Rev. D 63 (2001) 094006.

[7] V. M. Abazov et al. [D0 Collaboration], DØ Note 5089conf

[8] J. Campbell, F. Maltoni and F. Tramontano, Phys. Rev. Lett. 98 (2007) 252002.

[9] P. Artoisenet, J. P. Lansberg and F. Maltoni, Phys. Lett. B 653 (2007) 60.

[10] J. Pumplin, D. R. Stump, J. Huston, H. L. Lai, P. Nadolsky and W. K. Tung, JHEP 0207 (2002) 012

[11] V. M. Abazov et al. [D0 Collaboration], Phys. Rev. Lett. 94 (2005) 232001

[12] G. T. Bodwin, H. S. Chung, D. Kang, J. Lee and C. Yu, Phys. Rev. D 77 (2008) 094017.

[13] Ph. Hagler et al. Phys. Rev. D 63 (2001) 077501; F. Yuan and K. T. Chao, Phys. Rev. D 63 (2001) 034006.

[14] P. Artoisenet, F. Maltoni and T. Stelzer, JHEP 0802 (2008) 102.

[15] J. Alwall et al., Eur. Phys. J. C 53 (2008) 473.

[16] E. L. Berger, J. w. Qiu and Y. l. Wang, Phys. Rev. D 71 (2005) 034007

[17] B. Gong and J. X. Wang, arXiv:0802.3727 [hep-ph].

[18] B. Gong, X. Q. Li and J. X. Wang, arXiv:0805.4751 [hep$\mathrm{ph}$.

[19] H. Haberzettl and J. P. Lansberg, Phys. Rev. Lett. 100 (2008) 032006; J. P. Lansberg, J. R. Cudell and Yu. L. Kalinovsky, Phys. Lett. B 633 (2006) 301

[20] G. C. Nayak, J. W. Qiu and G. Sterman, Phys. Rev. Lett. 99 (2007) 212001 